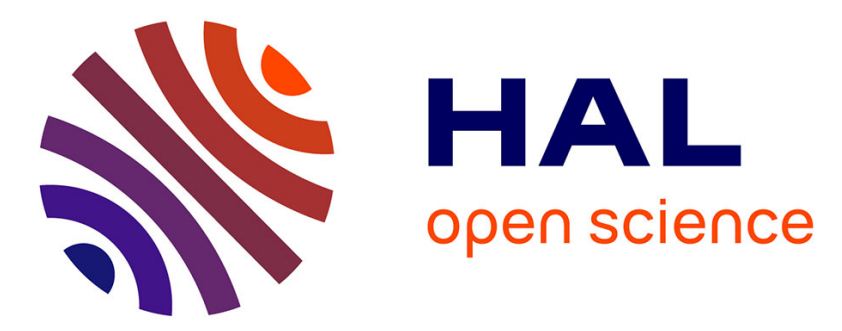

\title{
Transcriptomic analysis of untreated and drug-treated differentiated HepaRG cells over a 2-week period.
}

\author{
Camille C Savary, Xiaoqi Jiang, Marc Aubry, Rozenn Jossé, Annette
}

Kopp-Schneider, Philip Hewitt, André Guillouzo

\section{- To cite this version:}

Camille C Savary, Xiaoqi Jiang, Marc Aubry, Rozenn Jossé, Annette Kopp-Schneider, et al.. Transcriptomic analysis of untreated and drug-treated differentiated HepaRG cells over a 2-week period.. Toxicology in Vitro, 2015, 30 (1), pp.27-35. 10.1016/j.tiv.2014.12.019 . hal-01128182

HAL Id: hal-01128182

https://hal-univ-rennes1.archives-ouvertes.fr/hal-01128182

Submitted on 29 Apr 2015

HAL is a multi-disciplinary open access archive for the deposit and dissemination of scientific research documents, whether they are published or not. The documents may come from teaching and research institutions in France or abroad, or from public or private research centers.
L'archive ouverte pluridisciplinaire HAL, est destinée au dépôt et à la diffusion de documents scientifiques de niveau recherche, publiés ou non, émanant des établissements d'enseignement et de recherche français ou étrangers, des laboratoires publics ou privés. 


\section{Elsevier Editorial System(tm) for Toxicology in Vitro Manuscript Draft}

Manuscript Number:

Title: Transcriptomic analysis of untreated and drug-treated differentiated HepaRG cells over a 2-week period

Article Type: SI:PREDICT-IV report

Keywords: HepaRG cells; Transcriptomics; PPAR agonists; Repeated treatments; Time-dependent changes; gene profiling.

Corresponding Author: Dr. Camille Savary,

Corresponding Author's Institution: INSERM

First Author: Camille Savary

Order of Authors: Camille Savary; Xiaoqi Jiang; Marc Aubry; Rozenn Jossé; Annette Kopp-Schneider; Philip Hewitt; André Guillouzo

Abstract: Previous works have shown that differentiated HepaRG cells can exhibit drug metabolism activities close to those of primary human hepatocytes for several weeks at confluence. The present study was designed to evaluate their long-term functional stability and their response to repeated daily drug treatments over a 14-day period, using a transcriptomics approach. Our data show that less than $1 \%$ of the expressed genes were markedly deregulated over this period and mainly included downregulation of genes related to the cell cycle during the two weeks and overexpression of genes involved in xenobiotic and lipid metabolism from 3 days. After daily treatment with the three PPAR agonists, fenofibrate, troglitazone and rosiglitazone qualitative and/or quantitative changes in gene profiling were observed depending on the compound and duration of treatment. The highest increase in the number of deregulated genes as a function of drug treatment was seen with rosiglitazone. The most up-regulated genes common across the three compounds were mainly related to lipid and xenobiotic metabolisms. All the data support the conclusion that human HepaRG cells have unique functional stability at confluence and that they are suitable for investigations on chronic effects of drugs and other chemicals. 


\section{Transcriptomic analysis of untreated and drug-treated differentiated HepaRG cells over a 2-week period}

Camille C. Savary ${ }^{1,2}$, Xiaoqi Jiang ${ }^{3}$, Marc Aubry ${ }^{2,4}$, Rozenn Jossé ${ }^{1,2}$, Annette Kopp-

Schneider ${ }^{3}$, Philip Hewitt ${ }^{5}$, André Guillouzo ${ }^{1,2, a}$.

$5{ }^{1}$ Inserm U991, Faculté des Sciences Pharmaceutiques et Biologiques, Rennes, France

${ }^{2}$ Université de Rennes 1, France

${ }^{3}$ Department of Biostatistics, German Cancer Research Center (DKFZ), Heidelberg,

Germany

${ }^{4}$ CNRS UMR 6061 Genetic and Development, Rennes

$10{ }^{5}$ Non-Clinical Safety, Merck Serono, Merck KGaA, Darmstadt, Germany. 


\begin{abstract}
Previous works have shown that differentiated HepaRG cells can exhibit drug metabolism activities close to those of primary human hepatocytes for several weeks at confluence. The 15 present study was designed to evaluate their long-term functional stability and their response to repeated daily drug treatments over a 14-day period, using a transcriptomics approach. Our data show that less than $1 \%$ of the expressed genes were markedly deregulated over this period and mainly included down-regulation of genes related to the cell cycle during the two weeks and overexpression of genes involved in xenobiotic and lipid metabolism from 3 days. After daily treatment with the three PPAR agonists, fenofibrate, troglitazone and rosiglitazone qualitative and/or quantitative changes in gene profiling were observed depending on the compound and duration of treatment. The highest increase in the number of deregulated genes as a function of drug treatment was seen with rosiglitazone. The most up-regulated genes common across the three compounds were mainly related to lipid and xenobiotic metabolisms. All the data support the conclusion that human HepaRG cells have unique functional stability at confluence and that they are suitable for investigations on chronic effects of drugs and other chemicals.
\end{abstract}

Key words: HepaRG cells, Transcriptomics, PPAR agonists, Repeated treatments, Time-dependent changes, gene profiling

\begin{abstract}
Abbreviations:
CYP, cytochrome P450; DMSO, dimethyl sulfoxide ; FBS, fetal bovine serum; LFC, log2 fold change; PPAR, peroxisome proliferator-activated receptor.
\end{abstract}


The liver is the most complex and metabolically active organ in the human body; it performs hundreds of essential functions but is also affected by multiple diseases. The marked hepatic functional differences existing between human and laboratory animals have led to a growing interest for in vitro human liver cell preparations for investigations on normal and diseased 40 human liver (Guguen-Guillouzo and Guillouzo, 2010). Primary hepatocytes are the closest model to the liver in vivo (Guillouzo, 1998). However, they exhibit large inter-donor variability, as evidenced by analysis of basal and chemical-altered gene expression profiles in hepatocyte populations from multiple donors using transcriptomic approaches (Rogue et al., 2012). Indeed, it appears that, whatever the test chemical, only a very small set of 45 responsive genes is reproducibly altered among hepatocytes from multiple individual donors (Goyak et al., 2008; Rogue et al., 2012).

Liver cell lines are potential alternatives to primary hepatocytes. However, many studies have emphasized the poor resemblance between primary normal hepatocytes and liver cell lines, including their transcriptome (Boess et al., 2003; Hart et al., 2010; Jennen et al., 2010; 50 Olsavsky et al., 2007). Nevertheless, there is presently one exception represented by the HepaRG cell line that can differentiate from a bipotent progenitor state to attain features of normal adult hepatocytes in primary culture without losing the indefinite growth property of immortalized cell lines (Cerec et al., 2007; Guillouzo et al., 2007).

HepaRG cells have been shown to be a suitable in vitro cell model for studies on viral B infection and replication (Gripon et al., 2002) and xenobiotic metabolism and toxicity (Aninat et al., 2006; Antherieu et al., 2010; Antherieu et al., 2012). Recent studies have confirmed their resemblance to primary human hepatocytes by comparing their gene expression profiles either at the basal level or after chemical treatment using whole genome arrays, 
suggesting that HepaRG cells could be representative of primary human hepatocyte

populations (Lambert et al., 2009).

Contrary to other liver cell lines which continue to grow and consequently do not remain functionally stable, HepaRG cells cease to proliferate at confluence. Moreover, they do not form tumors in nude mice (Cerec et al., 2007). Importantly, HepaRG cells maintain their xenobiotic metabolism capacity, including their responsiveness to prototypical inducers, for

65 several weeks at confluence (Antherieu et al., 2010; Josse et al., 2008). However, their global long-term stability at the transcriptome level has not been evaluated yet.

Within the 5-year EU framework PREDICT-IV, we performed a detailed comparative analysis of the transcriptome of HepaRG cells after 1, 3 and 14 days in basal and drug-treated conditions using Illumina microarrays. Three PPARs agonists, fenofibrate, troglitazone and 70 rosiglitazone were used to evaluate drug-induced gene profiling changes related to repeated treatments. Our data show high reproducibility between different experiments, deregulation of few genes with time at confluence and some qualitative and quantitative changes in gene profiling depending on the PPAR agonist and the duration of treatment.

\section{Material and methods}

75 Reagents

Williams' E medium was supplied by Eurobio (Les Ulis, France) and fetal bovine serum (FBS) was from HyClone $\AA^{\circledR}$ (Thermo Fischer Scientific, Illkrich, France). Troglitazone and rosiglitazone were purchased from Calbiochem. Fenofibrate were from Sigma Aldrich (St. Quentin Fallavier, France). All other chemicals were of the highest quality available. 


\section{HepaRG cell cultures}

In the present study, HepaRG cells were first seeded at a density of $2.6 \times 10^{4} \mathrm{cells} / \mathrm{cm}^{2}$ in 6 well dishes in growth medium composed of Williams' E medium supplemented with $10 \%$ FBS, $2 \mathrm{mM}$ glutamine, 100 units $/ \mathrm{mL}$ penicillin, $100 \mu \mathrm{g} / \mathrm{mL}$ streptomycin and $5 \times 10^{-5} \mathrm{M}$ hydrocortisone hemisuccinate. After two weeks of culture, they were shifted to the same 85 culture medium supplemented with $2 \%$ dimethyl sulfoxide (DMSO) for two further weeks in order to reach maximum differentiation. At that time, differentiated HepaRG cell cultures were composed of both mature hepatocytes and primitive biliary epithelial cells (about 50\% of each type) (Cerec et al., 2007). The cells were issued from the same cryopreserved cell stock (passage 10) and used at passage 12.

\section{Drug treatments}

Two days before treatment, differentiated HepaRG cells were shifted to a medium containing $2 \%$ serum and $1 \%$ DMSO. In this medium CYP activities are still maintained at sufficient levels and protein binding of test drugs is usually limited. This medium was renewed daily. Cultures were treated with the PPAR alpha agonist fenofibrate, the two PPAR gamma 95 agonists troglitazone and rosiglitazone or the vehicule (DMSO) every day for up to 14 days (Fig 1). In preliminary experiments their cytotoxicity was assayed using the MTT test to select appropriate drug concentrations. A $10 \%$ toxic concentration $\left(\mathrm{TC}_{10}\right)$ was determined for each drug after 14-day repeated exposure and was used for all experiments, i.e. 30, 70 and 100 $\mu \mathrm{M}$ for troglitazone, rosiglitazone and fenofibrate respectively.

\section{RNA isolation.}

HepaRG cells were used after 1, 3 and 14 days for transcriptomic analysis. Briefly, they were washed with phosphate buffered saline (PBS) 1X and harvested in lysis buffer (RLT buffer and $\beta$-mercaptoethanol). Total RNA was isolated using the RNeasy mini Kit (Qiagen, Venlo, Netherlands). RNA quantity and purity were assessed with a Nanodrop ND-1000 
spectrophotometer (Nyxor Biotech, Paris, France) and RNA integrity was checked on a

\section{Microarray hybridizations.}

Gene expression analysis was performed using Illumina ${ }^{\circledR}$ HT 12 v3 BeadChip arrays (Illumina Inc.) allowing the analysis of $\sim 48,000$ transcripts. Synthesis of biotin-labelled cRNA 110 was performed in an automated procedure using a Theonyx Liquid Performer (Aviso $\mathrm{GmbH}$, ) and MessageAmp ${ }^{\mathrm{TM}}$ II aRNA amplification Kit (Ambion) with several modifications (Zidek et al., 2007). Instead of column cleanup, the bead-based Agencourt ${ }^{\circledR}$ RNAclean ${ }^{\mathrm{TM}}$ system $^{2}$ (Beckman Coulter) was applied to purify cDNA and cRNA. cRNA quantity was measured spectrophotometrically (NanoDrop®) and the 2100 Agilent Bio-Analyzer was used for quality assessment. Amplified biotinylated cRNA (750 ng) was hybridized onto the Illumina $\AA^{8}$ BeadChip in a Hybridization Cartridge under humidified conditions for $20 \mathrm{~h}$ at $58{ }^{\circ} \mathrm{C}$. The chips were then washed, stained for $10 \mathrm{~min}$ with $1 \mu \mathrm{g} / \mathrm{ml}$ streptavidin-conjugated Cy3 (Amersham Biosciences), and finally dried by centrifugation. Fluorescence detection was carried out by confocal laser scanning with the Illumina ${ }^{\circledR}$ BeadArray Reader (Illumina Inc) at $532 \mathrm{~nm}$ and $0.8 \mu \mathrm{m}$ resolution. Illumina $\AA^{\circledR}$ BeadStudio Software was used for condensing raw data and further to ensure array quality based on different control bead parameters as described in a previous study (Boehme et al., 2009).

\section{Data processing and statistical analysis}

Differences in gene expression measurements between corresponding time-matched vehicle 125 controls and drug treatment conditions were summarized for each feature as log2 fold change (LFC) value and associated p-value computed by application of the moderated (unpaired) t-test (Smyth, 2004). The Benjamini-Hochberg multiple testing procedure (Benjamini and Hochberg, 1995) was applied to correct p-values for multiple testing of features. The R package 'limma' (Smyth, 2005) was used for calculation. Differentially 
130 expressed genes were selected with the adjusted p-value of 0.01 and LFC cutoff of 0.58 (fold change cutoff of 1.5). Genes with a LFC value larger than 0.58 were regarded as upregulated while genes with a LFC value smaller than -0.58 were regarded as downregulated. For each time point, pathway hypotheses were generated using the Ingenuity Pathway Analysis $\AA^{\circledR}$ v.7.0 software (IPA, Ingenuity System, CA). The names of all 135 deregulated genes identified in the present study are given in the Supplemental Table 1.

\section{Results}

\section{Inter-assays correlation coefficients}

A total of 48,000 probes, corresponding to 25,000 annotated genes, were present on each 140 array. Seven independent experiments, each containing 2 to 8 technical duplicates, were performed over a 3-year period using cells derived from the same frozen cell stock at passage 12. Transcriptomes of differentiated cells maintained for 1,3 and 14 days at confluence were analyzed. Correlation coefficients were high, ranging from 0.98 to 0.99 for technical and biological replicates (data not shown). Using an arbitrary cut-off of 6 (on log2 145 data), the number of expressed genes varied between 12,791 and 13,536 and the lowest consistency did not fall below $88 \%$ (Fig. 2A, B).

Venn diagrams were drawn on the common expressed genes in all experiments; at the 3 time points the number of expressed genes was very close representing 13,026, 13,027 and 13,040 with 12,536 in common after 1,3 and 14 days respectively (Fig. 2C). Hierarchical 150 clustering showed that day 3 was closer to day 14 than to day 1 (Fig. 2C). Accordingly, principal component analysis did not allow to separate the 3 time points (Fig 2D). 


\section{Differential gene expression with time at confluence}

Differentially expressed genes of all the control samples are displayed in Figure 3 . The total number of deregulated genes was 106; among them 54 were different between day 1 and day 3; 71 between day 1 and day 14 (34\% up-regulated and 66\% down-regulated) and 36 between day 3 and day 14 (Fig. 3A). Taking into account only modulated genes, hierarchical clustering showed that day 1 was closer to day 3 than to day 14 (Fig. 3B).

Three major functional pathways differed as a function of culture time, i.e. cell cycle, lipid metabolism and xenobiotic metabolism; between days 1 and 3 most deregulated genes, were down-regulated while between days 3 and 14 a set of up-regulated genes was identified in addition (Fig. 3C). Taking day 1 as a reference, most genes involved in cell cycle were down-regulated after 3 or 14 days (Fig. 3C; Supplemental Fig. 1A) while many of the up-regulated genes after 14 days were involved in lipid and xenobiotic metabolisms (Fig. 3C; Supplemental Fig. 1B). Up-regulated genes related to lipid metabolism included THRSP, APO2, 4 and 5, HSD17B13, CYP4A11 and those related to xenobiotic metabolism CYP2A6, 2C18, 2C19, 3A4. Among other up-regulated genes were genes related to inflammation and stress (CRP, TMPRSS6, CD3D, INHBE), innate immunity (SKAP1, LEAP2) and transport (OSTalpha, SLC28A1, SLC7A9). The highest up- and down-regulated genes were THRSP (1.67 LFC corresponding to 3.2-fold change) and CDC20 (-1 LFC corresponding to 0.5-fold change) respectively.

\section{Influence of PPAR agonists on gene expression as a function of treatment duration}

In order to take into account time-related changes in gene expression profiles in untreated cultures changes in gene transcripts induced by the three PPAR agonists after 1, 3 and 14 days of treatment were estimated by comparison with corresponding controls. Total and 175 specific modulated genes are shown in Venn diagrams (Fig 4A). Thus, when considering the three time points a total of 99, 224 and 153 different genes with 20 genes in common was 
modulated by fenofibrate, rosiglitazone and troglitazone (Fig. 4A), representing 47, 39 and

15 commonly deregulated genes after 1,3 and 14 days of treatment to the three PPAR agonists respectively (Fig. 4C). Noticeably, more commonly deregulated genes were observed between the two PPARy agonists troglitazone and rosiglitazone (44) than between these two PPARy agonists and the PPARa agonist fenofibrate, representing 19 and 7 genes with troglitazone and rosiglitazone respectively (Fig. 4A). The number of up- and downregulated genes was variable for each drug at the different time points (Fig. 4B, Supplemental Table 2). The total number was higher on day 14 than on days 1-3 for 185 fenofibrate and especially rosiglitazone (Fig. 4B, Supplemental Table 2).

Using the Ingenuity Pathway Analysis ${ }^{\circledR}$ software we found that the major "top networks" included lipid metabolism for the three compounds and in addition, drug metabolism for rosiglitazone and fenofibrate. The top "tox lists" included fatty acid metabolism, xenobiotic metabolism signaling, PXR/RXR activation at the three time points and in addition, LPS/IL1 mediated inhibition of $R X R$ function at day 14 for rosiglitazone, $F X R / / R X R$ et PXR/RXR activation, LPS/IL1 mediated inhibition of RXR function at the three time points for troglitazone; fatty acid metabolism, PXR/RXR activation, LPS/IL1 mediated inhibition of RXR function, at the three time points for fenofibrate. Analysis of gene profiles changes induced by the three PPARs also showed that a number of modulated genes were related to lipid and xenobiotic metabolisms (Fig. 5).

Most deregulated genes by $100 \mu \mathrm{M}$ fenofibrate were up-regulated; they included genes involved in mitochondrial fatty acid oxidation and ketogenesis (CPT1A, HADHB, HADHB), peroxisomal $\beta$ oxidation (ACOX1, PEX11A), microsomal $\omega$ oxidation (CYP4A11), fatty acid binding and activation (FABP4), regulation of lipoprotein lipase (ANGPTL4), associated with lipid droplets (PLINs) and transcriptional factors (KLF10, KLF11) as well as with glucose/glycerol metabolism (PDK4). Down-regulated genes were only 13, 17 and 20 after 1, 
3 and 14 days of treatment; they included ADH4, GMNT and THRSP. Noteworthy, the number of deregulated genes increased only slightly between days 3 and 14 (81 versus 88); the main deregulated genes were found at the 3 time points and showed only limited variation, with the exception of THRSP which became repressed at day 3 and much more on day 14 (FLC: -2.6 versus -1.7 ).

The percentage of down-regulated genes with $70 \mu \mathrm{M}$ rosiglitazone and $30 \mu \mathrm{M}$ troglitazone was much higher than with fenofibrate, whatever the duration of treatment.

Various genes related to lipid metabolism including ANGPTL4, PLINs, PEX11A, DHRS9 and/or to xenobiotic metabolism (CYP3A4, UGT1A1) were also up-regulated with rosiglitazone including at day 14 and with troglitazone after 1-3 days of treatment. However, some major qualitative and quantitative differences were observed between the two glitazones. CYP1A1, CYP1B1 and CPT1 were up-regulated only by rosiglitazone, and CD36 only by troglitazone. GNMT and THRSP were down-regulated only by the latter. Several genes were down-regulated (PKLR, G6PC, ADH4) by the two glitazones. Noticeably; 15 out of the 16 genes found to be up-regulated by troglitazone at day 14; were already upregulated after 1 and/or 3 days. FABP4, the most overexpressed gene with fenofibrate was also the most overexpressed gene with troglitazone at the three time points as well as with rosiglitazone at 14 days, change being detected only at a $p$ value $>0.01$ at days 1 and 3 with the latter (Fig. 5, supplemental table 2).

\section{Discussion}

Although several studies have already dealt with transcriptomic analysis of HepaRG cells, either in the absence or after treatment with various drugs, all have been limited to a period not exceeding a few days (Hart et al., 2010; Lambert et al., 2009; Rogue et al., 2012). The present study showed that only few genes were deregulated in differentiated HepaRG cells for 2 weeks at confluence and that both qualitative and quantitative changes in gene 
expression were observed between the three PPAR agonists and for each of them, as a function of treatment duration.

The use of $1 \%$ DMSO and $2 \%$ serum was a compromise allowing daily drug treatments, long-term maintenance of sufficient activity levels of the CYPs, which are known to be sensitive to DMSO. The presence of $2 \%$ serum corresponding to $0.8-0.9 \mathrm{mg} / \mathrm{mL}$ albumin can result in binding of drugs to proteins (Kramer et al., 2012). However, biokinetics analysis of several drugs has shown only limited delay, if any, in their metabolism as observed with chlorpromazine (Broaders et al., 2014).

235 As found with Agilent microarrays (Rogue et al., 2012), the total number of expressed genes was very close from one experiment to another, using Illumina microarrays. The number of commonly expressed genes identified with the latter was slightly higher, i.e.12,536, $n=7$ versus $11,691, n=2$; this could be explained by the presence of genes encoding miRNA and LOC on Illumina arrays and/or the choice of different cut-off signals. Some probes can also be absent in either microarray, as for example UGT1A1 that was not found in the Agilent microarrays (Rogue et al., 2012).

The total number of deregulated genes with time in culture was very limited. Indeed, only 106 genes were found to be deregulated after 3 or 14 days, representing less than $1 \%$ of the total number of expressed genes. Such limited changes based on 7 independent experiments over a 3-year period bring further support to the high functional stability of the HepaRG cell line at confluence and to the strong inter-assay reproducibility of the data obtained with this cell line.

Importantly, most deregulated genes between days 1 and 3 were repressed and related to the cell cycle; they remained down-regulated thereafter. This could be explained by the absence of marked cell proliferation when the cells are differentiated and maintained at confluence. By contrast, most other deregulated genes between days 3 and 14 were 
overexpressed and related to xenobiotic and lipid metabolisms in agreement with previous

observations showing increase activity of several CYPs with time at confluence; e.g. CYP3A4, CYP2C9 and CYP1A2 (Antherieu et al., 2010; Josse et al., 2008). This increase 255 could be related to the presence of DMSO that is known to induce several major CYPs at high concentrations (Aninat et al., 2006).

Up to now, only short-term studies on drug-induced changes in gene profiling have been performed with HepaRG cells. In the current work we used three PPAR agonists, fenofibrate troglitazone and rosiglitazone, to evaluate the influence of repeated daily treatments. Since these compounds have already been investigated by the transcriptomics approach in HepaRG cells and/ or primary human hepatocytes over a short period of time (Rakhshandehroo et al., 2009) the results were used as references in the present study. Although different experimental conditions (medium composition, different microarrays) were employed here, the major target metabolic pathways and many deregulated genes were similar to those identified in previous reports with the same PPAR agonists. After daily treatments for 14 days lipid and drug metabolisms remained the two main deregulated pathways, as previously observed after a short term exposure (Rogue et al., 2011). However, both qualitative and quantitative changes in gene profiling were associated with repeated treatments. Thus, the number of deregulated genes was marked increased with fenofibrate and especially rosiglitazone, and amplification of up- or down-regulation of some genes was seen with each of the three PPAR agonists after a 2-week daily treatment.

Many PPARa target genes were found to be deregulated by fenofibrate. They included many genes related to lipid metabolism as well as other genes such as vinn-2 that is linked to inflammation and oxidative stress. Plasma vanin activity levels have been found to be increased significantly by fenofibrate in human subjects with type 2 diabetes (van Diepen et al., 2014). Recently, a map of the PPARa transcription regulatory network has been 
designed for primary human hepatocytes (McMullen et al., 2014). Noticeably many major PPARa target genes were similarly found to be deregulated in HepaRG cells, such as ACOX1, CPT1A, ANGPTL4, HMGC2, PLIN2 and SLC25A20. Our data are also in line with those reported in human hepatocytes treated with the PPARa agonist Wy14643 (Rakhshandehroo et al., 2009).

Treatments were glitazones also showed changes in gene profiling dependent on the compound and the duration of treatment. Thus, the high responsiveness of the CYP1 family to rosiglitazone after a short exposure (Rogue et al., 2011) was confirmed and shown to be 285 maintained with repeated treatments. Noteworthy, as with fenofibrate, FAPB4 was the highest up-regulated gene observed with troglitazone at the three time points, while it was not modulated with rosiglitazone at days 1 and 3 (at a pv <0.01). However it also became the most deregulated gene with this compound at day 14 . Similarly THRSP was the most or nearly the most repressed gene with fenofibrate and troglitazone while it was not modulated by rosiglitazone, except at 14 days. In addition, various qualitative and quantitative differences in gene expression levels were observed between the two glitazones in agreement with their quite different hepatotoxic potential. Interestingly, several genes previously reported to be modulated by the two glitazones in both primary human hepatocytes and HepaRG cells after a 24h treatment (Rogue et al., 2011) were also found to 295 be altered in the present study, e.g. ANGPTL4, CD36, PLIN4 and POR. Surprisingly, the number of modulated genes by troglitazone was much lower after 14 days than 1-3 days (47 versus 87-102); however, 15 out the 16 up-regulated genes were also found to be overexpressed after 1-3 days. Comparison of several concentrations of the compound and some qPCR analyses that are usually more sensitive than microarrays, should allow to better 300 understand in vitro long-term effects. 
Time-dependent quantitative changes were usually modest; those of FABP4 appearing as

the most prominent: indeed, its overexpression was markedly higher at day 3 and/ day 14 compared to day 1 with fenofibrate and troglitazone and its LFC reached 4.5 at day 14 versus no change at days 1-3 with rosiglitazone.

Another interesting observation was that many PPARa target genes, e.g. VNN1 and UGT1A1 (Senekeo-Effenberger et al., 2007), were also modulated by PPARy agonists,

In summary, our results show that changes in gene expression profiles in differentiated HepaRG cells are limited over a 2 weeks period and that daily treatments with PPAR agonists lead to some qualitative and quantitative changes related to the agonist and the 310 duration of treatment

\section{Acknowledgments}

This work was primarily supported by the European Union's Seventh Framework Programme (FP7/2007-2013), project Predict-IV, grant agreement 202222. We are grateful to Mrs Claudia Klement for microarrays preparation.

\section{References}

Aninat, C., Piton, A., Glaise, D., Le Charpentier, T., Langouet, S., Morel, F., Guguen-Guillouzo, C., Guillouzo, A., 2006. Expression of cytochromes P450, conjugating enzymes and nuclear receptors in human hepatoma HepaRG cells. Drug Metab Dispos 34, 75-83.

Antherieu, S., Chesne, C., Li, R., Camus, S., Lahoz, A., Picazo, L., Turpeinen, M., Tolonen, A., Uusitalo, J., Guguen-Guillouzo, C., Guillouzo, A., 2010. Stable expression, activity, and inducibility of cytochromes P450 in differentiated HepaRG cells. Drug Metab Dispos 38, 516-525.

Antherieu, S., Chesne, C., Li, R., Guguen-Guillouzo, C., Guillouzo, A., 2012. Optimization of the HepaRG cell model for drug metabolism and toxicity studies. Toxicol In Vitro 26, 1278-1285. 
Benjamini, Y., Hochberg, Y., 1995. Controlling the False Discovery Rate: A Practical and Powerful 325 Approach to Multiple Testing. Journal of the Royal Statistical Society. Series B (Methodological) 57, 289-300.

Boehme, K., Simon, S., Mueller, S.O., 2009. Gene expression profiling in Ishikawa cells: a fingerprint for estrogen active compounds. Toxicol Appl Pharmacol 236, 85-96.

Boess, F., Kamber, M., Romer, S., Gasser, R., Muller, D., Albertini, S., Suter, L., 2003. Gene expression in two hepatic cell lines, cultured primary hepatocytes, and liver slices compared to the in vivo liver gene expression in rats: possible implications for toxicogenomics use of in vitro systems. Toxicol Sci $73,386-402$.

Broeders J. J.W., Parmentier C.,Truisi, G. L., Jossé, R., Alexandre, E., Savary, C.C., Hewitt, P. G., Mueller, S. O., Guillouzo, A., Richert ' L., van Eijkeren, J. C.H., Hermens, J. L.M, Blaauboer, B.J.,2014. 335 Biokinetics of chlorpromazine in primary rat and human hepatocytes and human HepaRG cells after repeated exposure. Toxicol in Vitro, in press.

Cerec, V., Glaise, D., Garnier, D., Morosan, S., Turlin, B., Drenou, B., Gripon, P., Kremsdorf, D., Guguen-Guillouzo, C., Corlu, A., 2007. Transdifferentiation of hepatocyte-like cells from the human hepatoma HepaRG cell line through bipotent progenitor. Hepatology 45, 957-967.

Goyak, K.M., Johnson, M.C., Strom, S.C., Omiecinski, C.J., 2008. Expression profiling of interindividual variability following xenobiotic exposures in primary human hepatocyte cultures. Toxicol Appl Pharmacol 231, 216-224. 
Gripon, P., Rumin, S., Urban, S., Le Seyec, J., Glaise, D., Cannie, I., Guyomard, C., Lucas, J., Trepo, C.,

Guguen-Guillouzo, C., 2002. Infection of a human hepatoma cell line by hepatitis B virus. Proc Natl Acad Sci U S A 99, 15655-15660.

Guguen-Guillouzo, C., Guillouzo, A., 2010. General review on in vitro hepatocyte models and their applications. Methods Mol Biol 640, 1-40.

Guillouzo, A., 1998. Liver cell models in in vitro toxicology. Environ Health Perspect 106 Suppl 2, 511532.

Guillouzo, A., Corlu, A., Aninat, C., Glaise, D., Morel, F., Guguen-Guillouzo, C., 2007. The human hepatoma HepaRG cells: a highly differentiated model for studies of liver metabolism and toxicity of xenobiotics. Chem Biol Interact 168, 66-73.

Hart, S.N., Li, Y., Nakamoto, K., Subileau, E.A., Steen, D., Zhong, X.B., 2010. A comparison of whole genome gene expression profiles of HepaRG cells and HepG2 cells to primary human hepatocytes and human liver tissues. Drug Metab Dispos 38, 988-994.

Jennen, D.G., Magkoufopoulou, C., Ketelslegers, H.B., van Herwijnen, M.H., Kleinjans, J.C., van Delft, J.H., 2010. Comparison of HepG2 and HepaRG by whole-genome gene expression analysis for the purpose of chemical hazard identification. Toxicol Sci 115, 66-79.

Josse, R., Aninat, C., Glaise, D., Dumont, J., Fessard, V., Morel, F., Poul, J.M., Guguen-Guillouzo, C., Guillouzo, A., 2008. Long-term functional stability of human HepaRG hepatocytes and use for chronic toxicity and genotoxicity studies. Drug Metab Dispos 36, 1111-1118.

Kramer, N.I., Krismartina, M., Rico-Rico, A., Blaauboer, B.J., Hermens, J.L., 2012. Quantifying processes determining the free concentration of phenanthrene in Basal cytotoxicity assays. Chem Res Toxicol 25, 436-445. 
Lambert, C.B., Spire, C., Claude, N., Guillouzo, A., 2009. Dose- and time-dependent effects of phenobarbital on gene expression profiling in human hepatoma HepaRG cells. Toxicol Appl Pharmacol 234, 345-360.

McMullen, P.D., Bhattacharya, S., Woods, C.G., Sun, B., Yarborough, K., Ross, S.M., Miller, M.E., 370 McBride, M.T., LeCluyse, E.L., Clewell, R.A., Andersen, M.E., 2014. A map of the PPARalpha transcription regulatory network for primary human hepatocytes. Chem Biol Interact 209, 14-24.

Olsavsky, K.M., Page, J.L., Johnson, M.C., Zarbl, H., Strom, S.C., Omiecinski, C.J., 2007. Gene expression profiling and differentiation assessment in primary human hepatocyte cultures, established hepatoma cell lines, and human liver tissues. Toxicol Appl Pharmacol 222, 42-56.

375 Rakhshandehroo, M., Hooiveld, G., Muller, M., Kersten, S., 2009. Comparative analysis of gene regulation by the transcription factor PPARalpha between mouse and human. PLoS One 4, e6796.

Rogue, A., Lambert, C., Spire, C., Claude, N., Guillouzo, A., 2012. Interindividual variability in gene expression profiles in human hepatocytes and comparison with HepaRG cells. Drug Metab Dispos 40, 151-158.

380 Rogue, A., Lambert, C.B., Josse, R., Antherieu, S., Spire, C., Claude, N., Guillouzo, A., 2011. Comparative gene expression profiles induced by PPARY and PPAR $\alpha / \gamma$ agonists in human hepatocytes. PLoS One 6:e18816.

Senekeo-Effenberger, K., Chen, S., Brace-Sinnokrak, E., Bonzo, J.A., Yueh, M.F., Argikar, U., Kaeding, J., Trottier, J., Remmel, R.P., Ritter, J.K., Barbier, O., Tukey, R.H., 2007. Expression of the human UGT1 385 locus in transgenic mice by 4-chloro-6-(2,3-xylidino)-2-pyrimidinylthioacetic acid (WY-14643) and implications on drug metabolism through peroxisome proliferator-activated receptor alpha activation. Drug Metab Dispos 35, 419-427. 
Smyth, G.K., 2004. Linear models and empirical bayes methods for assessing differential expression in microarray experiments. Stat Appl Genet Mol Biol 3, 3.

Smyth, G.K., 2005. Limma: linear models for microarray data, Bioinformatics and computational biology solutions using R and Bioconductor. Springer, pp. 397-420.

van Diepen, J.A., Jansen, P.A., Ballak, D.B., Hijmans, A., Hooiveld, G.J., Rommelaere, S., Galland, F., Naquet, P., Rutjes, F.P., Mensink, R.P., Schrauwen, P., Tack, C.J., Netea, M.G., Kersten, S., Schalkwijk, J., Stienstra, R., 2014. PPAR-alpha dependent regulation of vanin-1 mediates hepatic lipid metabolism. J Hepatol 61, 366-372.

Zidek, N., Hellmann, J., Kramer, P.J., Hewitt, P.G., 2007. Acute hepatotoxicity: a predictive model based on focused illumina microarrays. Toxicological Sciences 99, 289-302. 


\section{Figure legends}

Figure 1: Schematic representation of the experimental design. Differentiated HepaRG cells were prepared as previously described (Cerec et al., 2007): after seeding at low density, they were allowed to proliferate and at confluence were shifted to a medium containing FBS and $2 \%$ DMSO for 2 weeks. At that time, the medium was changed with medium containing 2\% FBS and 1\% DMSO for daily exposure. After 2 days, experiments started: cells were exposed to PPAR agonists daily for 14 days. mRNAs were prepared after 1,3 and 14 days.

Figure 2: Expressed genes in different experiments after 1, 3 and 14 days. Numbers of expressed genes in different experiments (A); Percentage of commonly expressed genes between experiments (B); Hierarchical clustering and Venn diagrams of expressed genes as a function of time at confluence taking into account all experiments $(\mathrm{C})$; Principal component analysis representing all experiments (D).

Figure 3: Effects of culture time on gene expression in HepaRG cells. Number of differentially expressed genes between 1,3 and 14 days $(A)$. Heat map taking into account modulated genes $(F C \geq 1.5, \leq-1.5$ with $p v \leq 0.01)(B)$. Heat map taking first day as a reference of modulated genes involved in cell cycle, lipid and drug metabolisms (C).

Figure 4: Effects of PPAR agonist exposure on HepaRG transcriptome after 1, 3 and 14 days. Venn diagrams depicting the differentially expressed genes between drugs; three days are grouped (A); Number of up and down-deregulated genes by each drug (B); Venn diagrams depicting the differentially expressed genes between 1, 3 and 14 days of exposure to fenofibrate, rosiglitazone and troglitazone (C).

Figure 5: Top networks affected as a function of time by PPAR agonists. These networks were identified by Ingenuity Pathway Analysis® software. The actual values $(\log 2$ 
fold change) are shown as a heatmap, where red represents increased and green decreased

1 expression respectively. Modulated genes, LFC $>0.58$ or $<-0.58, p v<0.01$ at least for 1 condition (days of treatment). 
Figure 1

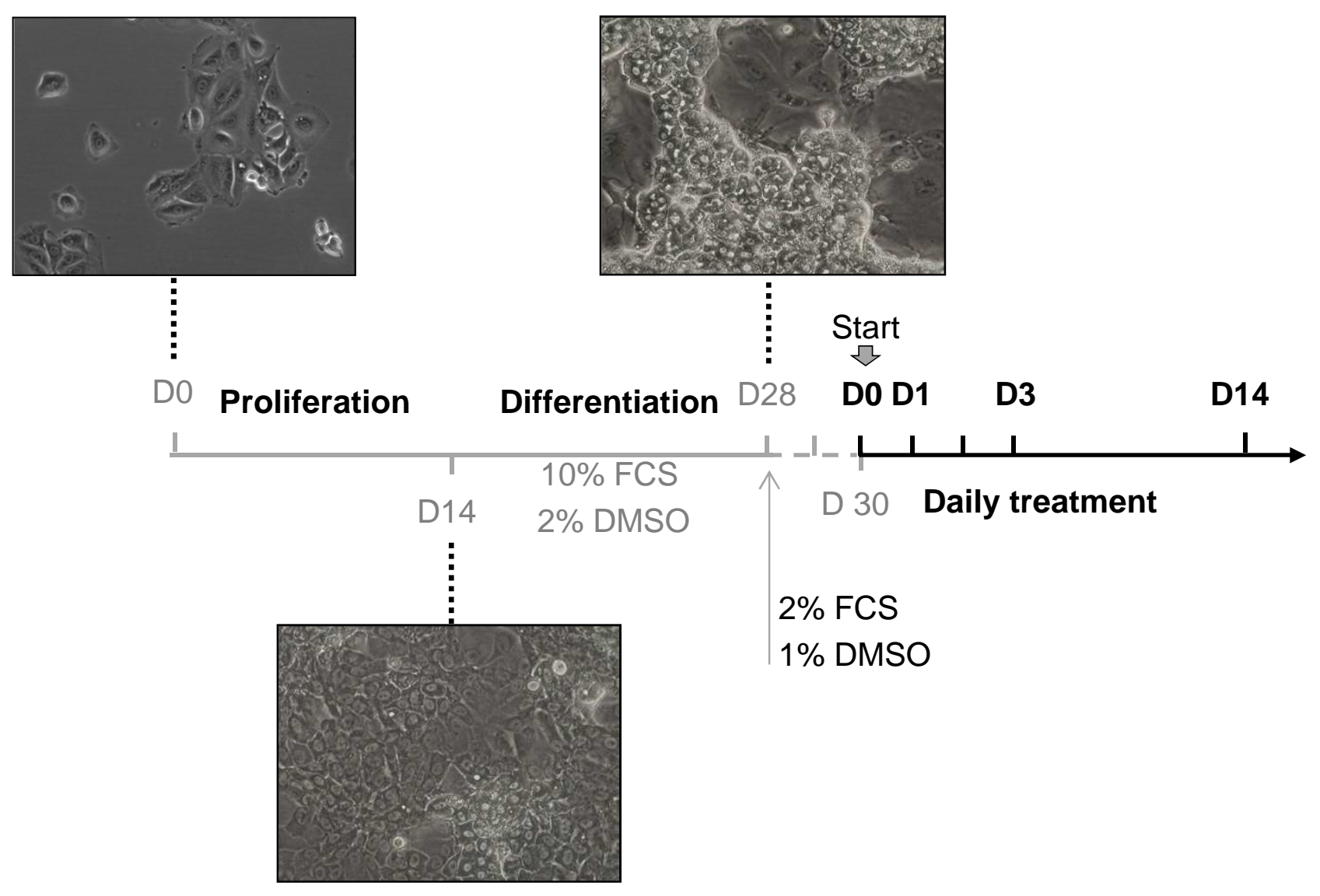


Figure 2

A

\begin{tabular}{ccccccccc} 
experiment & $\mathbf{1}$ & $\mathbf{2}$ & $\mathbf{3}$ & $\mathbf{4}$ & $\mathbf{5}$ & $\mathbf{6}$ & $\mathbf{7}$ & Total \\
\hline D1 & 13395 & 13053 & 13478 & 12810 & 13124 & 13501 & 13321 & 13026 \\
D3 & 13399 & 12977 & 13437 & 12801 & 13114 & 13533 & 13373 & 13017 \\
D14 & 13299 & 13016 & 13536 & 12791 & 13108 & 13510 & 13292 & 13040 \\
\hline
\end{tabular}

B

\begin{tabular}{rrrrrrrr}
$\mathrm{D} 1$ & 1 & \multicolumn{1}{c}{2} & \multicolumn{1}{c}{3} & \multicolumn{1}{c}{4} & \multicolumn{1}{c}{5} & \multicolumn{1}{c}{6} & \multicolumn{1}{c}{7} \\
\hline 1 & 100 & 94 & 94 & 91 & 93 & 92 & 92 \\
2 & 96 & 100 & 95 & 92 & 95 & 93 & 94 \\
3 & 93 & 92 & 100 & 92 & 93 & 93 & 94 \\
4 & 95 & 94 & 96 & 100 & 96 & 97 & 96 \\
5 & 95 & 94 & 96 & 93 & 100 & 95 & 96 \\
6 & 91 & 90 & 93 & 92 & 93 & 100 & 94 \\
7 & 93 & 92 & 95 & 92 & 94 & 96 & 100 \\
\hline
\end{tabular}

\begin{tabular}{lrrrrrrr}
$\mathrm{D} 3$ & 1 & 2 & 3 & 4 & 5 & 6 & 7 \\
\hline 1 & 100 & 93 & 94 & 89 & 92 & 92 & 92 \\
2 & 96 & 100 & 95 & 91 & 94 & 93 & 94 \\
3 & 93 & 92 & 100 & 91 & 93 & 94 & 94 \\
4 & 93 & 92 & 95 & 100 & 94 & 95 & 94 \\
5 & 94 & 93 & 96 & 91 & 100 & 96 & 96 \\
6 & 91 & 89 & 93 & 89 & 93 & 100 & 94 \\
7 & 92 & 91 & 94 & 90 & 94 & 95 & 100 \\
\hline
\end{tabular}

\begin{tabular}{llllllll}
$\mathrm{D} 14$ & 1 & 2 & 3 & 4 & 5 & 6 & 7 \\
\hline 1 & 100 & 93 & 96 & 90 & 95 & 94 & 94 \\
2 & 95 & 100 & 97 & 90 & 95 & 93 & 94 \\
3 & 94 & 93 & 100 & 88 & 94 & 92 & 93 \\
4 & 94 & 91 & 93 & 100 & 93 & 95 & 93 \\
5 & 96 & 95 & 97 & 91 & 100 & 95 & 96 \\
6 & 92 & 90 & 92 & 90 & 92 & 100 & 94 \\
7 & 94 & 92 & 94 & 90 & 94 & 95 & 100 \\
\hline
\end{tabular}

C

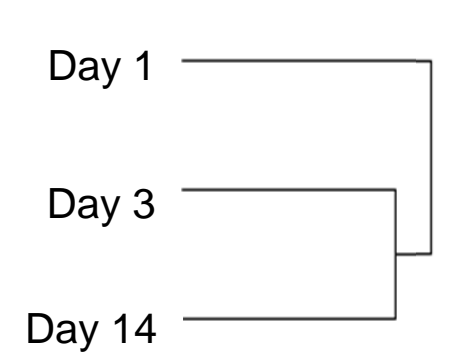

3026 gen 1

Day 3

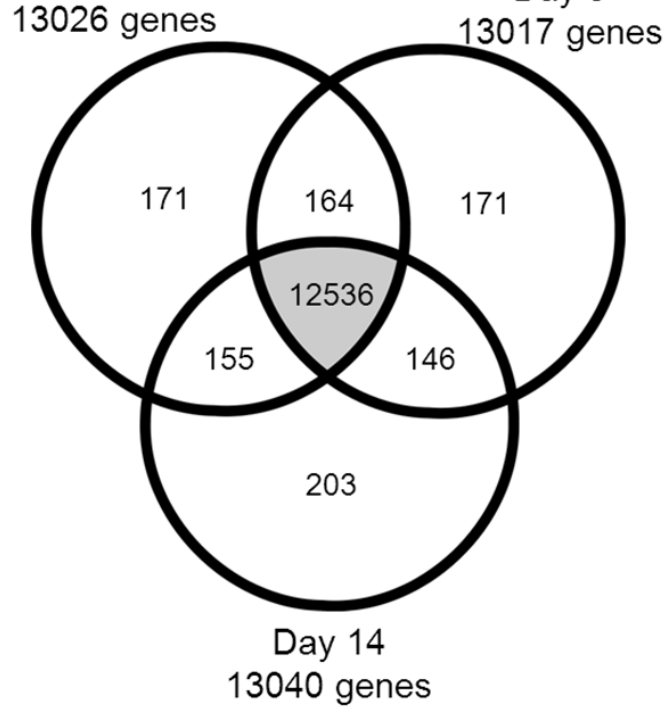

D

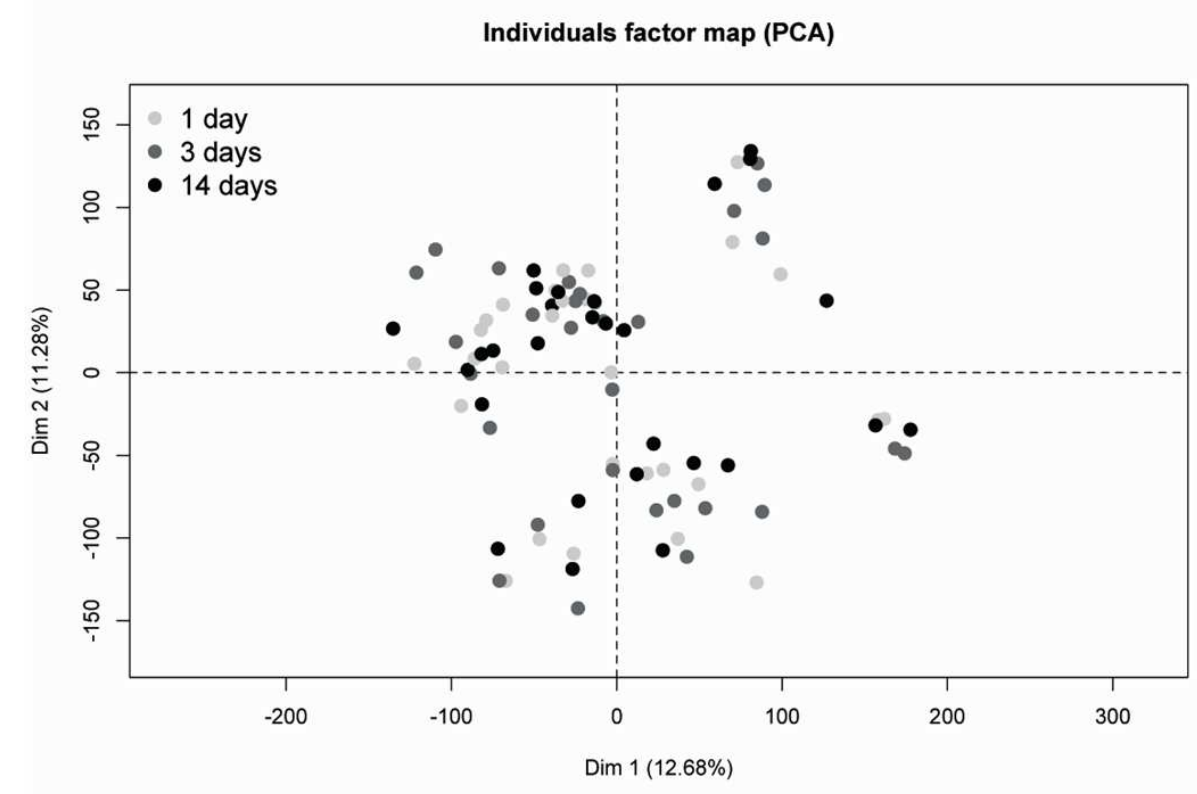


Figure 3

\begin{tabular}{ccccc}
$A$ & $\begin{array}{c}\text { D1 vs } \\
\text { D3 }\end{array}$ & $\begin{array}{c}\text { D1 vs } \\
\text { D14 }\end{array}$ & $\begin{array}{c}\text { D3 vs } \\
\text { D14 }\end{array}$ & $\begin{array}{c}\text { D1+D3 } \\
\text { +D14 }\end{array}$ \\
\hline 54 & 71 & 36 & 106 \\
\hline
\end{tabular}

B

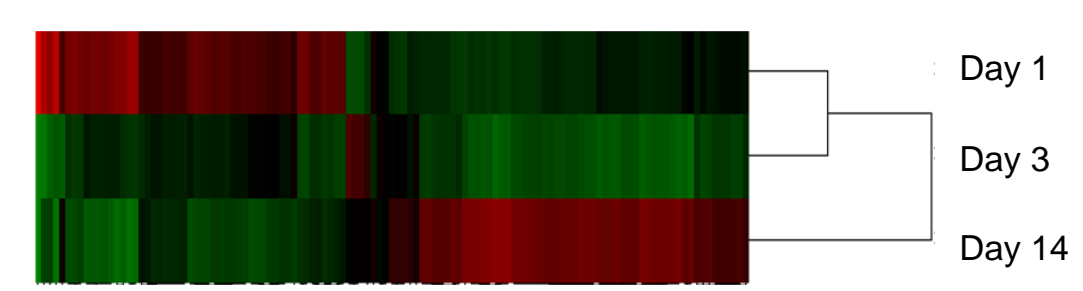

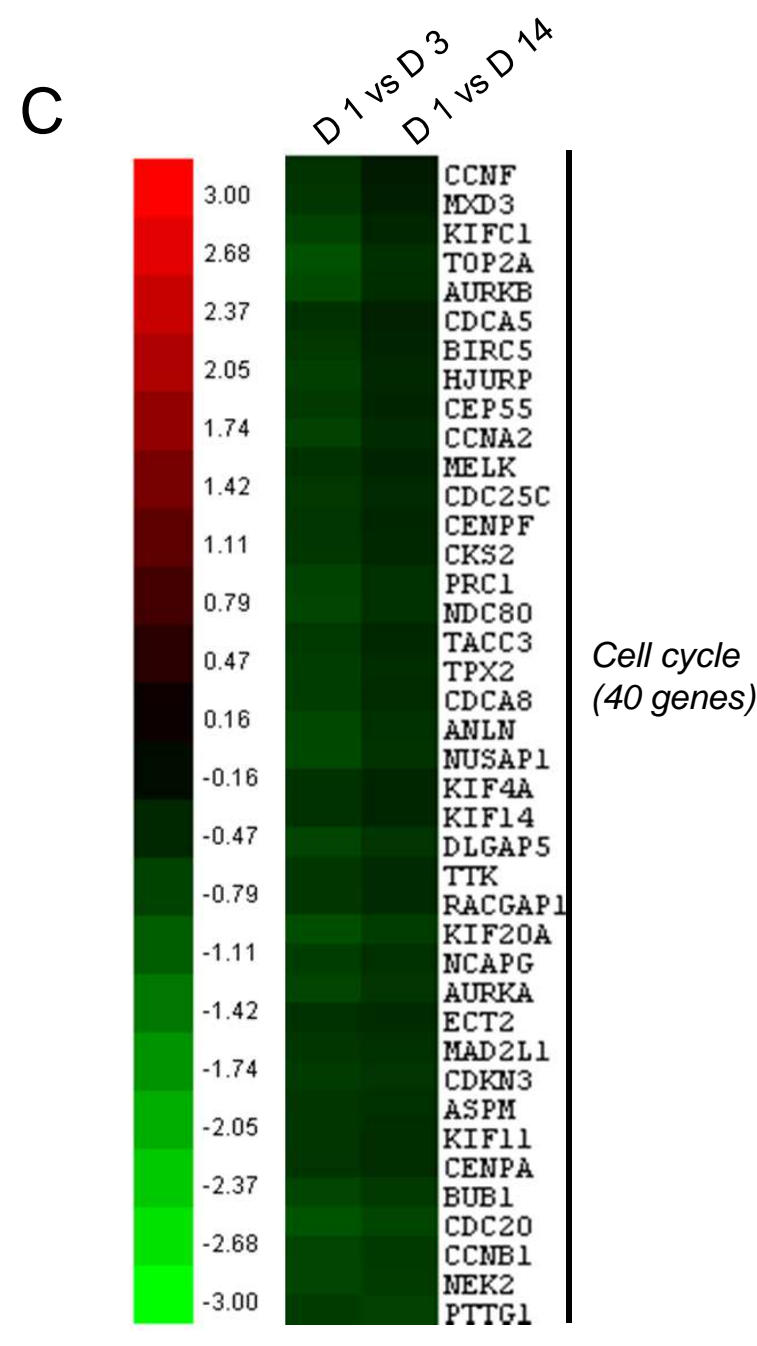


Figure 4

A

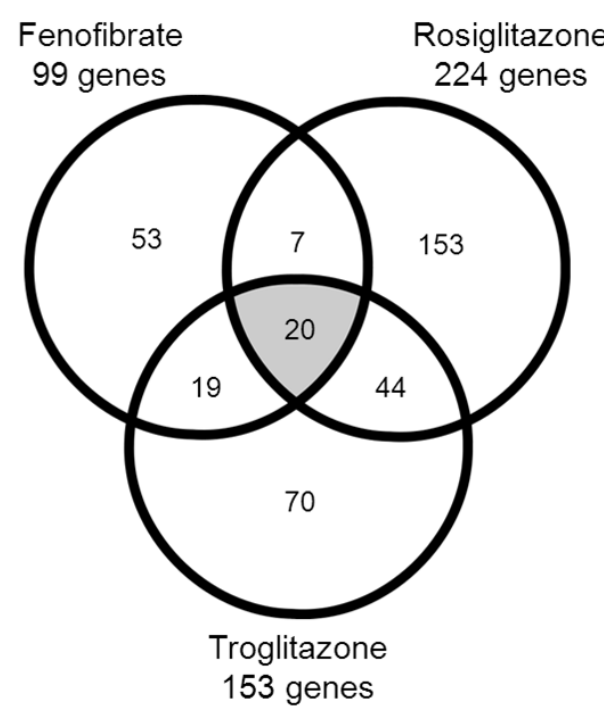

C

Fenofibrate

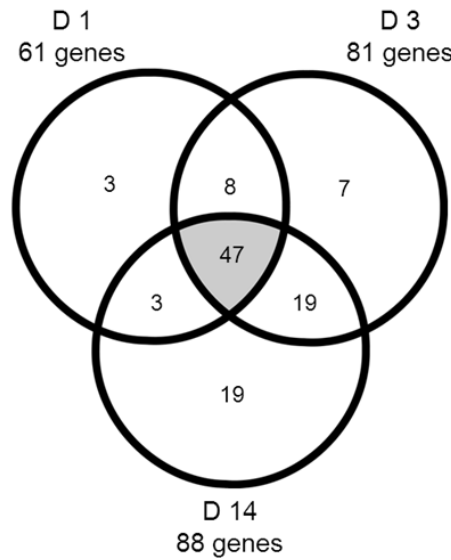

B

\begin{tabular}{|c|cc|ccc|cc|}
\hline & \multicolumn{2}{c}{ Fenofobrate } & \multicolumn{2}{c}{ Rosiglitazone } & \multicolumn{2}{c|}{ Troglitazone } \\
& UP & DOWN & UP & DOWN & UP & DOWN \\
\hline D1 & 48 & 13 & 56 & 78 & 24 & 60 \\
D3 & 64 & 17 & 19 & 44 & 45 & 57 \\
D14 & 66 & 20 & 47 & 139 & 16 & 31 \\
\hline
\end{tabular}

Rosiglitazone

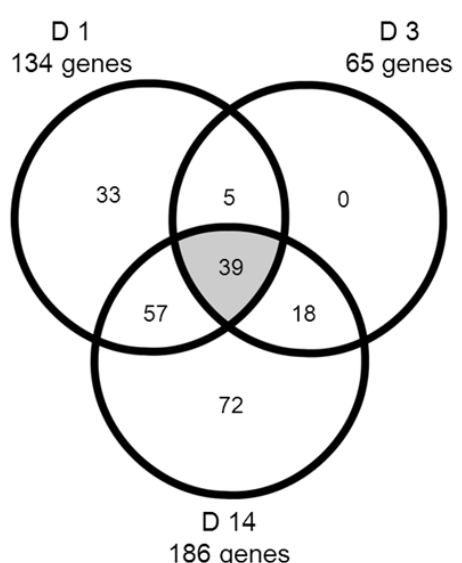

Troglitazone

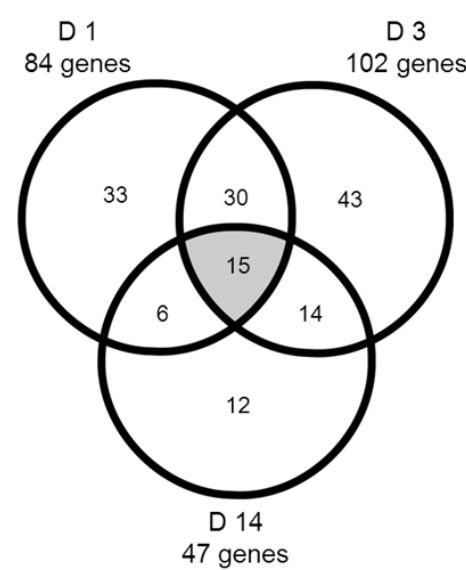


Figure 5
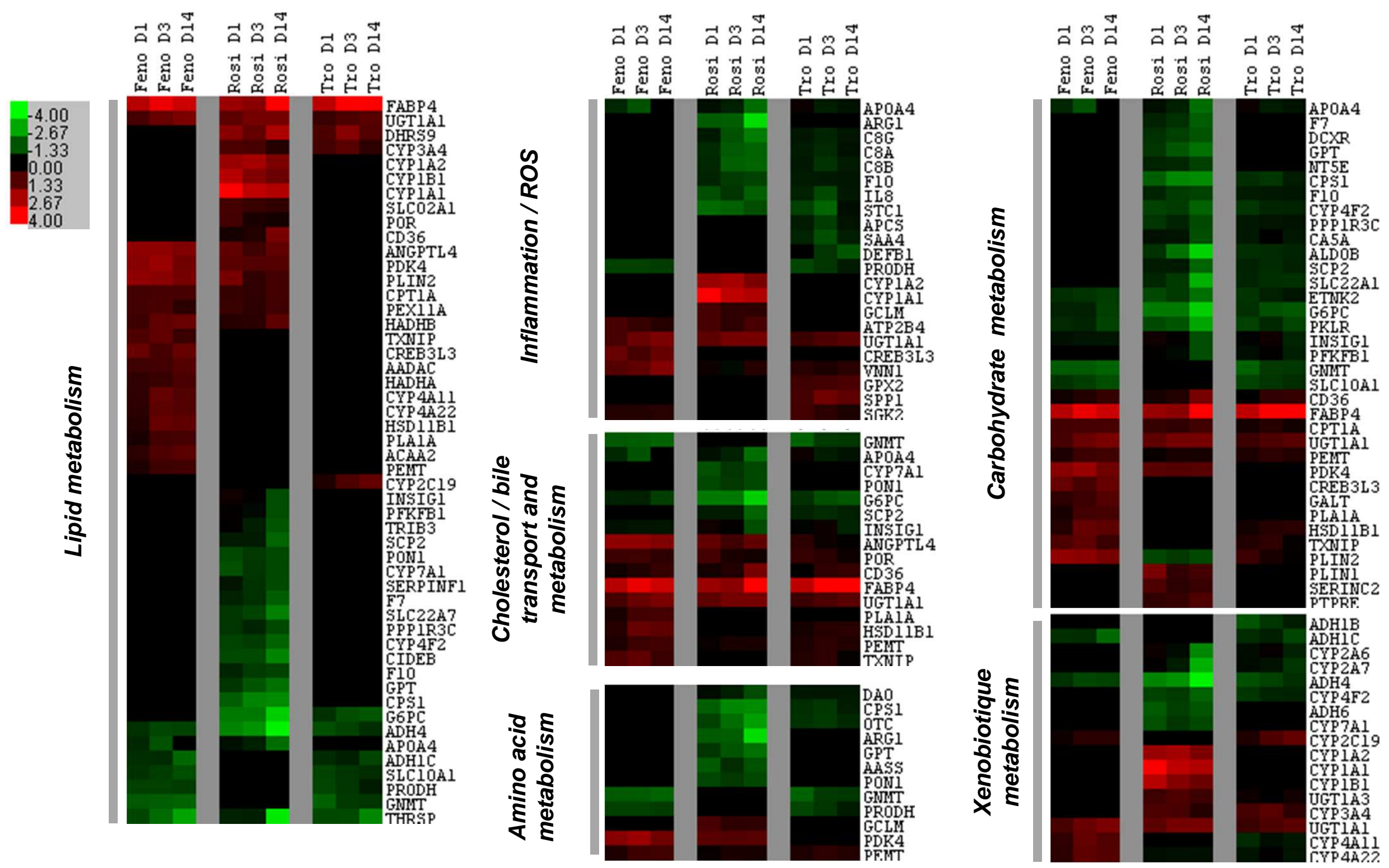
Supplementary Material
Click here to download Supplementary Material: Supplemental data TIV.pdf

Supplementary Material
Click here to download Supplementary Material: Supplemental data TIV.pdf

Cut

(1)

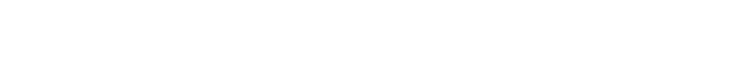

$\sqrt{10}$

.

(1)

(1)

(1)

(1)

.

.

.

.

.

.

.

.

.

.

.

.

.

.

.

.

.

.

.

.

.

.

.

.

.

.

.

.

. 
${ }^{\star}$ Conflict of Interest
Click here to downlo

Click here to download Conflict of Interest: Savary coi_disclosure.pdf 
*Conflict of Interest
Click here to downloa

Click here to download Conflict of Interest: Jiang coi_disclosure.pdf

\section{Click here to download}


*Conflict of Interest
Click here to downloa

Click here to download Conflict of Interest: Aubry coi_disclosure.pdf

\section{Click here to download}


Click here to download Conflict of Interest: Joss coi_disclosure.pdf 
*Conflict of Interest
Click here to download Conflict of Interest: Kopp-Schneider coi_disclosure.pdf

*Conflict of Interest
Click here to download Conflict of Interest: Kopp-Schneider coi_disclosure.pdf

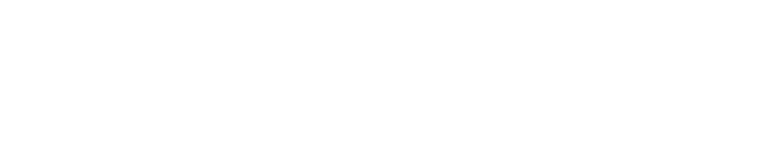

Click here to download Conflict of Interest: Kopp-Schneider coi_disclosure.pdf

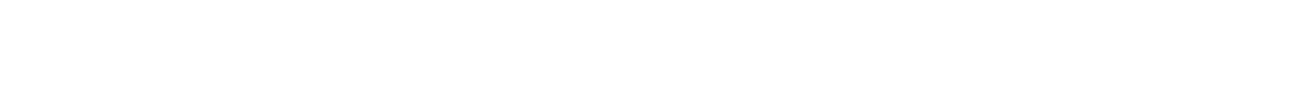

$\left(\frac{10}{10}\right.$

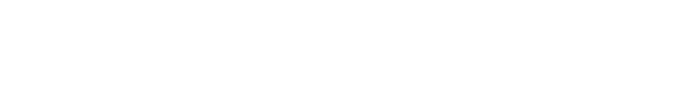

(1)

(1)

(1)

(1)

(1)

(1)

.

.

.

.

.

.

.

.

.

.

.

.

.

.

.

.

.

.

.

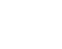
(1) 
Conflict of Interest

Click here to download Conflict of Interest: Hewitt coi_disclosure.pdf 
*Conflict of Interest
Click here to downloa

Click here to download Conflict of Interest: Guillouzo coi_disclosure.pdf 